\title{
Imaging nanoscale Fermi-surface variations in an inhomogeneous superconductor
}

\author{
W. D. Wise ${ }^{1}$, Kamalesh Chatterjee ${ }^{1}$, M. C. Boyer ${ }^{1}$, Takeshi Kondo ${ }^{1,2 \star}$, T. Takeuchi, ${ }^{2,3}$, H. Ikuta $^{2}$, \\ Zhijun $\mathrm{Xu}^{4}$, Jinsheng Wen ${ }^{4}$, G. D. Gu${ }^{4}$, Yayu Wang ${ }^{1 \star}$ and E. W. Hudson ${ }^{1 \dagger}$
}

\begin{abstract}
Particle-wave duality suggests we think of electrons as waves stretched across a sample, with wavevector $k$ proportional to their momentum. Their arrangement in 'k-space', and in particular the shape of the Fermi surface, where the highest-energy electrons of the system reside, determine many material properties. Here we use a novel extension of Fourier-transform scanning tunnelling microscopy to probe the Fermi surface of the strongly inhomogeneous Bi-based cuprate superconductors. Surprisingly, we find that, rather than being globally defined, the Fermi surface changes on nanometre length scales. Just as shifting tide lines expose variations of water height, changing Fermi surfaces indicate strong local doping variations. This discovery, unprecedented in any material, paves the way for an understanding of other inhomogeneous characteristics of the cuprates, such as the pseudogap magnitude, and highlights a new approach to the study of nanoscale inhomogeneity in general.
\end{abstract}

That high-temperature superconductors should show nanoscale inhomogeneity is unsurprising. In correlated electron materials, Coulomb repulsion between electrons hinders the formation of a homogeneous Fermi liquid, and complex real-space phase separation is ubiquitous ${ }^{1}$. Scanning tunnelling microscopy (STM) measurements have revealed significant spectral variations in a number of cuprates including $\mathrm{Bi}_{2} \mathrm{Sr}_{2} \mathrm{CuO}_{6+x}$ (Bi-2201; ref. 2) and $\mathrm{Bi}_{2} \mathrm{Sr}_{2} \mathrm{CaCu}_{2} \mathrm{O}_{8+x}$ (Bi-2212; refs 3-5).

This intrinsic inhomogeneity poses challenges to the interpretation of bulk or spatially averaged measurements. For example, angle-resolved photoemission spectroscopy (ARPES) is a powerful technique for studying $\mathbf{k}$-space structure in the cuprates ${ }^{6}$. However, ARPES can provide only spatially averaged results, and uniting these with the nanoscale disordered electronic structure measured by STM remains a formidable task.

Our approach to addressing this issue originates from discoveries by Fourier-transform STM (FT-STM), which has emerged as an important tool for studying the cuprates. These studies begin with the collection of a spectral survey, in which differential conductance spectra, proportional to local density of states (LDOS), are measured at a dense array of locations, creating a three-dimensional dataset of LDOS as a function of energy and position in the plane. By Fourier transforming constant-energy slices of these surveys, referred to as LDOS or conductance maps, FT-STM enables the study of two phenomena linked to the cuprate Fermi surface (FS) (Fig. 1b). First, non-dispersive wavevectors of the checkerboardlike charge order observed in many cuprates ${ }^{7-10}$ are probably connected to the FS-nesting wavevectors near the antinodal $(\pi, 0)$ Brillouin zone boundary (see, for example, the arrow in Fig. 1b $)^{11}$. Second, dispersive quasiparticle interference (QPI) patterns ${ }^{12-14}$ originate from elastic scattering of quasiparticles on the FS near the $\operatorname{nodal}(\pi, \pi)$ direction $^{15}$. Taken together, these phenomena provide complementary information about the cuprate FS.

However, because these phenomena were previously characterized using Fourier transforms of large LDOS maps containing a wide range of energy gaps and spectra, previous FT-STM mapping of the FS was still spatially averaged ${ }^{16}$. The atomic-scale spatial resolution of STM was not exploited, so connections between FS geometry and local electronic structure went unexamined.

Here we introduce two new STM analysis techniques, which enable extraction of a local FS. In studies of Bi-2201 and Bi-2212, we find that the cuprate FS varies at the nanometre scale, and that its local geometry correlates strongly with the size of the large, inhomogeneous energy gap that has been extensively studied by STM (ref. 4) and which we associate with the pseudogap ${ }^{2}$.

We first investigate the spatial dependence of the antinodal FS using checkerboard charge order. Our recent study of Bi-2201 showed that the average checkerboard wavevector decreases with increased doping ${ }^{11}$. This trend, inconsistent with many proposed explanations of the checkerboard, matches the doping dependence of the antinodal FS-nesting wavevector (Fig. 1b), and led us to conclude that the checkerboard is caused by a FS-nesting induced charge-density wave. Here we continue the investigation of the three Bi-2201 dopings considered in our previous work, two underdoped with superconducting transitions at $25 \mathrm{~K}$ (UD25) and $32 \mathrm{~K}$ (UD32), and one optimally doped with $T_{\mathrm{c}}=35 \mathrm{~K}$ (OP35). Although, following convention, we previously reported FT-measured, spatially averaged wavevectors, careful observation of the checkerboard pattern (Fig. 2a) shows that the periodicity changes drastically with position.

One way of analysing this variation is with a Voronoi diagram. After identifying local peaks of the checkerboard modulation (local maxima in the $+10 \mathrm{mV}$ conductance map, identified as red dots in Fig. 2a), we divide the map into cells, each containing points closer to one checkerboard maximum than any other. The square root of the cell size is a measure of the local checkerboard wavelength. We find that this local wavelength is highly correlated with the previously observed gap-size inhomogeneity (Fig. 2b,c), with a correlation coefficient of -0.4 .

Another method of investigating this relationship between local checkerboard periodicity and gap size is to modify the traditional

\footnotetext{
${ }^{1}$ Department of Physics, Massachusetts Institute of Technology, Cambridge, Massachusetts 02139, USA, ${ }^{2}$ Department of Crystalline Materials Science, Nagoya University, Nagoya 464-8603, Japan, ${ }^{3}$ EcoTopia Science Institute, Nagoya University, Nagoya 464-8603, Japan, ${ }^{4}$ Condensed Matter Physics and Materials Sciences Department, Brookhaven National Laboratory, Upton, New York 11973, USA. *Present addresses: Ames Laboratory and Dept. of Physics and Astronomy, lowa State University, Ames, lowa 50011, USA (T.K.); Department of Physics, Tsinghua University, Beijing 100084, China (Y.W.).

†e-mail: ehudson@mit.edu.
} 

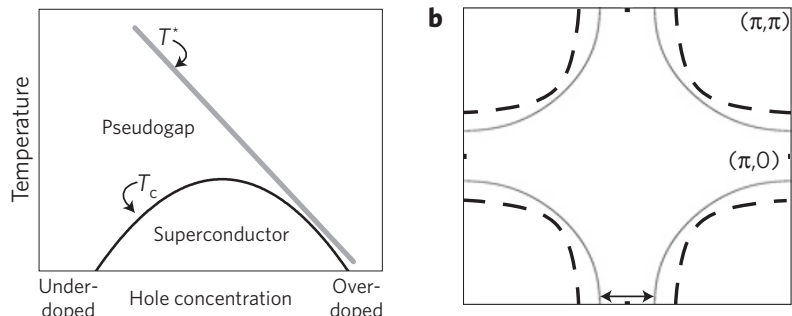

Figure 1 | Phase diagram and FS topology of the cuprates. a, A minimal generic phase diagram of the high-temperature superconductors shows a superconducting transition temperature $T_{c}$ that is parabolic with doping, peaking at optimal doping, whereas the pseudogap temperature $T^{*}$-and the proportional pseudogap magnitude $\Delta_{\mathrm{PG}}$-decrease nearly linearly with doping. b. The hole-doped cuprate FS is typically seen as hole-like, closing around empty states centred at $(\pi, \pi)$, rather than filled states centred at $(0,0)$. Moving from optimally doped (solid line) to underdoped (dashed) materials, the hole pockets shrink. This increases the length of the nesting vector (arrow) near the antinode.

FT technique by first masking the LDOS map by gap size. This technique is illustrated in Fig. 3. The LDOS map is set to zero everywhere outside a desired gap range and then Fourier transformed to reveal a gap-dependent checkerboard wavevector. The result is qualitatively similar to the Fourier transform of the complete map, but the wavevector measured is due solely to the fraction of the sample within the selected range of gap magnitudes. Fourier transforms of different regions reveal different wavevectors (Fig. 3b,c). Consistent with previously reported sample averages ${ }^{11}$, wavevectors increase with gap size (Fig. 3d). We note that this trend is not an artefact of mask geometry; rotating the masks, which preserves their geometry while eliminating their relation to the gap map, eliminates the trend of Fig. 3d, simply yielding instead the sample average wavevector for all maps (see Supplementary Information).

These two independent techniques not only demonstrate the inhomogeneity of the checkerboard wavelength, the likely cause of universally reported short checkerboard correlation lengths ${ }^{11}$, but also reveal that the checkerboard wavevector and local gap size are strongly correlated. Between samples, average checkerboard wavevector decreases with increased doping ${ }^{11}$, consistent with the decrease of the antinodal FS-nesting wavevector (Fig. 1b). The tunnelling-measured gap size (scaling with pseudogap temperature $T^{*}$ of Fig. 1a) also on average decreases with increased doping. Thus the positive correlation of local gap size and checkerboard wavevector is consistent with a picture in which local FS variations, driven by local doping variations, affect both. Notably, where gap sizes from different samples overlap, so do their checkerboard wavelengths (Fig. 3d), indicating that checkerboard properties are truly set by local rather than sample average properties. We stress that this result is independent of the cause of the checkerboard, and relies only on our previous report of its doping dependence ${ }^{11}$.

To further investigate this idea we next turn to QPI studies of slightly overdoped $\left(T_{\mathrm{c}}=89 \mathrm{~K}\right) \mathrm{Bi}-2212$. The idea behind QPI, pioneered by the Davis group ${ }^{12-14}$ and Dung-Hai Lee ${ }^{15}$, is illustrated in Fig. 4a. Interference patterns arising from quasiparticle scattering are dominated by wavevectors connecting $\mathbf{k}$-space points of high density of states. For any given energy, eight such symmetric points exist, all on the FS. The well-defined wavevectors (coloured lines) of the resultant interference pattern can therefore be used to reconstruct the FS.

Just as in checkerboard studies, previous work on QPI has yielded spatially averaged results ${ }^{12-14}$. As above, we extend QPI analysis to yield local, gap-dependent information. The interference wavevectors (circled in Fig. 4b) found in Fourier transforms of
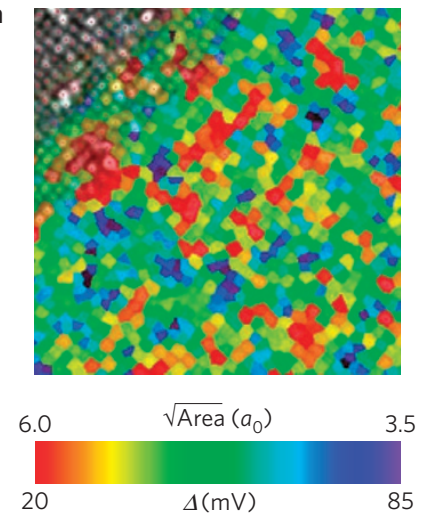

b

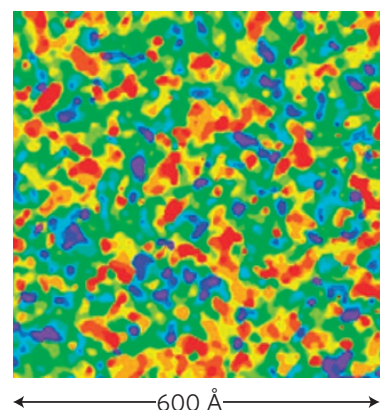

Figure 2 | Local variations of the Bi-2201 checkerboard. a, Conductance map (energy $E=+10 \mathrm{mV}$ slice of a 400 pixel, $600 \AA$ spectral survey) of $T_{\mathrm{C}}=32 \mathrm{~K}$ underdoped $\mathrm{Bi}-2201$, showing a spatially varying checkerboard charge modulation (upper left). Voronoi cells, associated with

checkerboard maxima (red dots) and coloured to indicate their area, enable determination of local wavelength. $\mathbf{b}$, Traditional gap map of the same area showing well known variations of gap size $\Delta$. c, Spectra from the survey (sorted, averaged and coloured by gap size, and shifted vertically for clarity) highlight the remarkable low-energy homogeneity in the presence of strong higher-energy inhomogeneity. Spectral survey parameters: $I_{\text {set }}=400 \mathrm{pA}, V_{\text {sample }}=-200 \mathrm{mV}$

gap-masked conductance maps can be inverted to derive an FS (ref. 13), now associated with the gap range of the mask. Doing this for two different gap ranges, from 30 to $60 \mathrm{mV}(\langle\Delta\rangle=37 \mathrm{mV})$, and 10 to $30 \mathrm{mV}(\langle\Delta\rangle=26 \mathrm{mV})$, we find distinct shifts in the FS (Fig. 4c). We extend this to the antinode with checkerboard order, resolvable in Bi-2212 as non-dispersive order at energies above the point where the QPI signal weakens. Adding nested antinodal FS segments (dashed lines) derived from checkerboard periodicity, we arrive at a nearly complete view of different local FSs corresponding to different spatial locations, correlated spatially with different gap sizes. We also plot in Fig. 4c rigid-band tight-binding FSs (ref. 17) from two different dopings ( $p=0.10$ and 0.18 as calculated from the pocket area) very similar to the surfaces we derive.

Although $\mathbf{k}$-space variation on nanometre length scales may at first glance seem shocking, on further reflection this result is not entirely surprising. Raising or lowering a uniformly slanted sea floor near the shore (changing the amount of sea above the floor) changes the position of the shoreline. Analogously, raising or lowering local doping changes the local FS. It has even been demonstrated that the locations of dopant oxygen atoms correlate with local gap-size variations ${ }^{18}$.

Interestingly, the correlation found in ref. 18 was the opposite of what we might at first expect from a local doping picture. While oxygen dopants contribute holes and hence increase the global doping of the sample, they correlate with an increased local gap size, consistent with underdoping. This led the authors and others ${ }^{19}$ to 

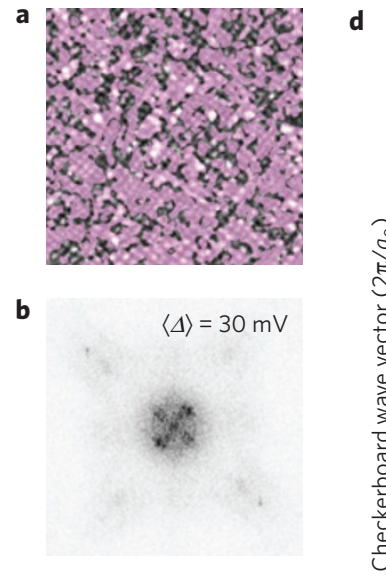

$$
\langle\Delta\rangle=30 \mathrm{mV}
$$

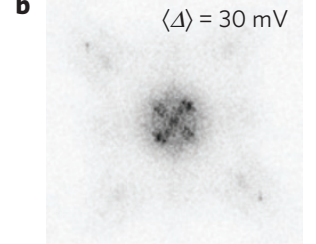

C $\langle\Delta\rangle=60 \mathrm{mV}$

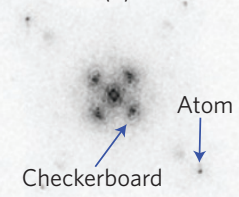

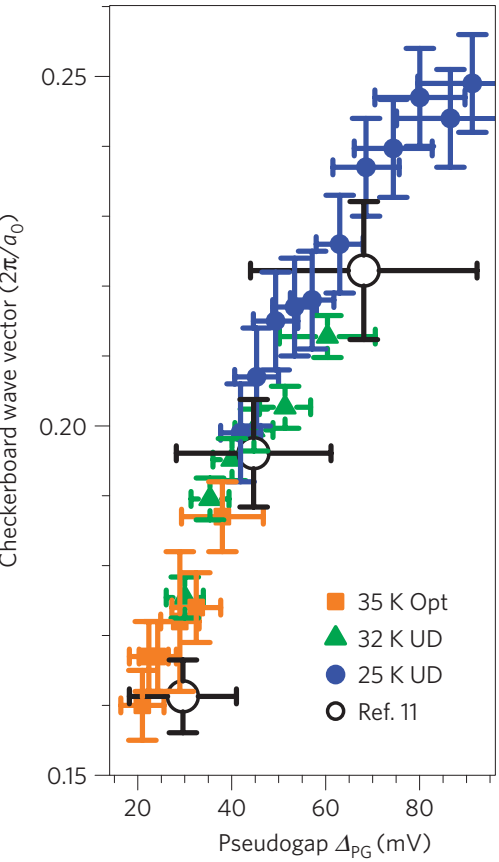

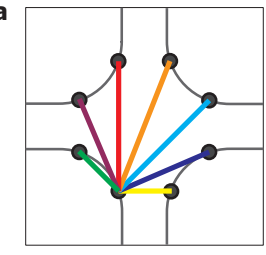

b

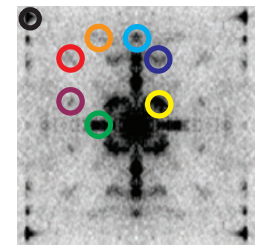

c

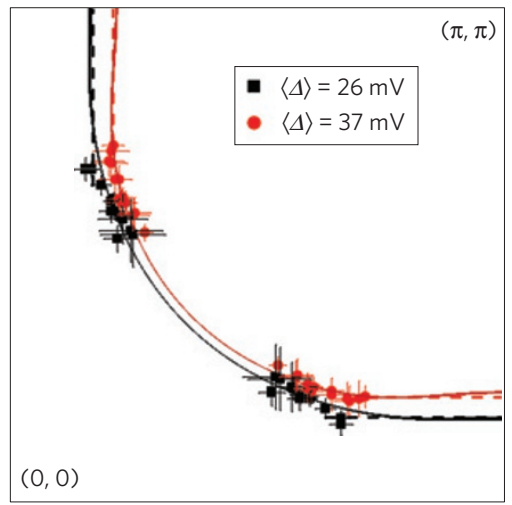

Figure 4 | QPI-derived local FS in Bi-2212. a, A schematic diagram of the FS (solid line) in the first Brillouin zone, showing symmetry, leading to an eightfold replication of any points at which the density of states peaks (for example circles). Scattering between quasiparticles at these points leads to a set of interference wavevectors (coloured lines), corresponding to peaks in interference maps such as $\mathbf{b}$, a Fourier transform of a $600 \AA$, 400 pixel, $E=12 \mathrm{mV}$ conductance map of $89 \mathrm{~K}$ overdoped $\mathrm{Bi}-2212$. The positions of these peaks (defined in terms of the atomic wavevector circled in black) uniquely define a position in $\mathbf{k}$-space on the FS. Fitting interference peaks in a series of fast Fourier transform maps at various energies from two different masks of the same data leads to c, two different 'local FSs' (solid symbols with error bars indicating standard deviation for values obtained from different interference peaks). Dashed lines, obtained from

checkerboard-determined nesting wavevectors, extend the determined FS to the antinode. Solid lines are FSs from a rigid-band, tight-binding model ${ }^{17}$ at two different dopings, $p=0.10$ and 0.18 .

Coupled with the observation that the width of the gap (and hence doping) distribution scales with mean gap size ${ }^{24}$, and is thus smaller in overdoped than underdoped samples, inhomogeneity should have a stronger effect on ARPES measurements in underdoped than in overdoped samples. This effect is particularly apparent in $\mathrm{Bi}-2201$, which is more inhomogeneous than Bi-2212 (ref. 2).

Despite the success of this interpretation, some outstanding questions remain. The model curves ${ }^{17}$ of Fig. $4 \mathrm{c}$ suggest that the effective band energy may shift by as much as $20 \mathrm{mV}$ between different regions of the sample. This shift would lead to strong scattering, even in the nodal direction. However, aside from the reasonable match to our extracted FSs, there is no reason to believe that this global average-extracted rigid-band model should completely describe the local FSs. For example, our extracted FSs seem closer in the nodal region than the model surfaces.

Another question concerns a homogeneous gap we have reported ${ }^{2}$. The large, inhomogeneous gap discussed throughout this paper is probably more accurately termed the pseudogap, whereas we identified as the superconducting gap a second, relatively homogeneous smaller gap, which opens at $T_{c}$. One might imagine that superconductivity, as characterized by size of the superconducting gap, should be as strongly affected by inhomogeneous local doping as the pseudogap. This is not what we have observed ${ }^{2}$. One explanation is that doping-dependence differences make inhomogeneity affect the pseudogap more than the superconducting gap (the pseudogap, scaling with $T^{*}$, changes more than the superconducting gap, scaling with $T_{\mathrm{c}}$ ). Another explanation may lie in their momentum-space distribution. Raman spectroscopy $^{25}$ and ARPES (refs 26,27) results indicate that the superconducting gap is most strongly associated with near-nodal states, whereas the pseudogap arises near the antinodes. As noted above, the nodal region is significantly more homogeneous than the antinodal region, and hence could lead to more homogeneous superconducting than pseudogap properties. 
This interpretation also points towards an explanation of bulk measurement results. Although several are suggestive of nanoscale inhomogeneity ${ }^{4}$, including neutron measurements of the magnetic resonance peak width ${ }^{28}$, thermodynamic measurements seem inconsistent with strong inhomogeneity ${ }^{29}$. These measurements, however, are most sensitive to the nature of the superconducting gap and the low-energy density of states, both of which seem homogeneous. Undoubtedly, these homogeneous properties relate to the homogeneity of the nearnodal FS. Nonetheless, they are remarkable given the strong inhomogeneity we report here.

Inhomogeneity of doping or charge is common in many materials, and leads naturally to the idea of nanoscale FS variation. This work is, to our knowledge, the first attempt to characterize these variations, and raises the question of whether an FS, typically thought of as a bulk property, can be meaningfully defined inside nanometre-sized domains. Although our experimental results seem consistent with this picture, further experimental and theoretical work is needed to determine at what point a $\mathbf{k}$-space description such as this stops being useful.

\section{Received 15 July 2008; accepted 23 December 2008; published online 25 January 2009}

\section{References}

1. Dagotto, E. Complexity in strongly correlated electronic systems. Science 309, 257-262 (2005).

2. Boyer, M. C. et al. Imaging the two gaps of the high-temperature superconductor $\mathrm{Bi}_{2} \mathrm{Sr}_{2} \mathrm{CuO}_{6+x}$. Nature Phys. 3, 802-806 (2007).

3. Howald, C., Fournier, P. \& Kapitulnik, A. Inherent inhomogeneities in tunneling spectra of $\mathrm{Bi}_{2} \mathrm{Sr}_{2} \mathrm{CaCu}_{2} \mathrm{O}_{8-x}$ crystals in the superconducting state. Phys. Rev. B 64, 100504 (2001).

4. Lang, K. M. et al. Imaging the granular structure of high- $T_{\mathrm{C}}$ superconductivity in underdoped $\mathrm{Bi}_{2} \mathrm{Sr}_{2} \mathrm{CaCu}_{2} \mathrm{O}_{8+\delta}$. Nature 415, 412-416 (2002).

5. Gomes, K. K. et al. Visualizing pair formation on the atomic scale in the high- $T_{\mathrm{c}}$ superconductor $\mathrm{Bi}_{2} \mathrm{Sr}_{2} \mathrm{CaCu}_{2} \mathrm{O}_{8+\delta}$. Nature 447, 569-572 (2007).

6. Damascelli, A., Hussain, Z. \& Shen, Z.-X. Angle-resolved photoemission studies of the cuprate superconductors. Rev. Mod. Phys. 75, 473-541 (2003).

7. Howald, C., Eisaki, H., Kaneko, N., Greven, M. \& Kapitulnik, A. Periodic density-of-states modulations in superconducting $\mathrm{Bi}_{2} \mathrm{Sr}_{2} \mathrm{CaCu}_{2} \mathrm{O}_{8+\delta}$. Phys. Rev. B 67, 014533 (2003).

8. Vershinin, M. et al. Local ordering in the pseudogap state of the high- $T_{\mathrm{C}}$ superconductor $\mathrm{Bi}_{2} \mathrm{Sr}_{2} \mathrm{CaCu}_{2} \mathrm{O}_{8+\delta}$. Science 303, 1995-1998 (2004).

9. Hanaguri, T. et al. A 'checkerboard' electronic crystal state in lightly hole-doped $\mathrm{Ca}_{2-x} \mathrm{Na}_{x} \mathrm{CuO}_{2} \mathrm{Cl}_{2}$. Nature 430, 1001-1005 (2004).

10. McElroy, K. et al. Coincidence of checkerboard charge order and antinodal state decoherence in strongly underdoped superconducting $\mathrm{Bi}_{2} \mathrm{Sr}_{2} \mathrm{CaCu}_{2} \mathrm{O}_{8+\delta}$. Phys. Rev. Lett. 94, 197005 (2005).

11. Wise, W. D. et al. Charge-density-wave origin of cuprate checkerboard visualized by scanning tunnelling microscopy. Nature Phys. 4, 696-699 (2008).

12. Hoffman, J. E. et al. Imaging quasiparticle interference in $\mathrm{Bi}_{2} \mathrm{Sr}_{2} \mathrm{CaCu}_{2} \mathrm{O}_{8+\delta}$. Science 297, 1148-1151 (2002).

13. McElroy, K. et al. Relating atomic-scale electronic phenomena to wave-like quasiparticle states in superconducting $\mathrm{Bi}_{2} \mathrm{Sr}_{2} \mathrm{CaCu}_{2} \mathrm{O}_{8+\delta}$. Nature 422, 592-596 (2003).

14. Hanaguri, T. et al. Quasiparticle interference and superconducting gap in $\mathrm{Ca}_{2-x} \mathrm{Na}_{x} \mathrm{CuO}_{2} \mathrm{Cl}_{2}$. Nature Phys. 3, 865-871 (2007).
15. Wang, Q.-H. \& Lee, D.-H. Quasiparticle scattering interference in high-temperature superconductors. Phys. Rev. B 67, 020511 (2003).

16. Del Maestro, A., Rosenow, B. \& Sachdev, S. From stripe to checkerboard ordering of charge-density waves on the square lattice in the presence of quenched disorder. Phys. Rev. B 74, 024520 (2006).

17. Norman, M. R., Randeria, M., Ding, H. \& Campuzano, J. C. Phenomenological models for the gap anisotropy of $\mathrm{Bi}_{2} \mathrm{Sr}_{2} \mathrm{CaCu}_{2} \mathrm{O}_{8}$ as measured by angle-resolved photoemission spectroscopy. Phys. Rev. B 52, 615-622 (1995).

18. McElroy, K. et al. Atomic-scale sources and mechanism of nanoscale electronic disorder in $\mathrm{Bi}_{2} \mathrm{Sr}_{2} \mathrm{CaCu}_{2} \mathrm{O}_{8+\delta}$. Science 309, 1048-1052 (2005).

19. Nunner, T. S., Andersen, B. M., Melikyan, A. \& Hirschfeld, P. J. Dopant-modulated pair interaction in cuprate superconductors. Phys. Rev. Lett. 95, 177003 (2005).

20. Zhou, S., Ding, H. \& Wang, Z. Correlating off-stoichiometric doping and nanoscale electronic inhomogeneity in the high- $T_{\mathrm{c}}$ superconductor $\mathrm{Bi}_{2} \mathrm{Sr}_{2} \mathrm{CaCu}_{2} \mathrm{O}_{8+\delta}$. Phys. Rev. Lett. 98, 076401 (2007).

21. Slezak, J. A. et al. Imaging the impact on cuprate superconductivity of varying the interatomic distances within individual crystal unit cells. Proc. Natl Acad. Sci. 105, 3203-3208 (2008).

22. Pasupathy, A. N. et al. Electronic origin of the inhomogeneous pairing interaction in the high- $T_{\mathrm{c}}$ superconductor $\mathrm{Bi}_{2} \mathrm{Sr}_{2} \mathrm{CaCu}_{2} \mathrm{O}_{8+\delta}$. Science 320, 196-201 (2008).

23. Zhou, X. J. et al. Dichotomy between nodal and antinodal quasiparticles in underdoped $\left(\mathrm{La}_{2-x} \mathrm{Sr}_{x}\right) \mathrm{CuO}_{4}$ superconductors. Phys. Rev. Lett. 92, 187001 (2004).

24. Alldredge, J. W. et al. Evolution of the electronic excitation spectrum with strongly diminishing hole density in superconducting $\mathrm{Bi}_{2} \mathrm{Sr}_{2} \mathrm{CaCu}_{2} \mathrm{O}_{8+\delta}$. Nature Phys. 4, 319-326 (2008).

25. Le Tacon, M. et al. Two energy scales and two distinct quasiparticle dynamics in the superconducting state of underdoped cuprates. Nature Phys. 2, 537-543 (2006).

26. Tanaka, K. et al. Distinct Fermi-momentum-dependent energy gaps in deeply underdoped Bi2212. Science 314, 1910-1913 (2006).

27. Kondo, T., Takeuchi, T., Kaminski, A., Tsuda, S. \& Shin, S. Evidence for two energy scales in the superconducting state of optimally doped $(\mathrm{Bi}, \mathrm{Pb})_{2}(\mathrm{Sr}, \mathrm{La})_{2} \mathrm{CuO}_{6+\delta}$. Phys. Rev. Lett. 98, 267004 (2007).

28. Fauqué, B. et al. Dispersion of the odd magnetic resonant mode in near-optimally doped $\mathrm{Bi}_{2} \mathrm{Sr}_{2} \mathrm{CaCu}_{2} \mathrm{O}_{8+\delta}$. Phys. Rev. B 76, 214512 (2007).

29. Loram, J. W. \& Tallon, J. L. Thermodynamic transitions in inhomogeneous cuprate superconductors. Preprint at $<$ http://arxiv.org/abs/cond-mat/0609305>.

\section{Acknowledgements}

We thank A. V. Balatsky, N. Gedik, J. E. Hoffman, K. M. Lang, P. A. Lee, Y. Lee, T. Senthil and Z. Wang for comments. This research was supported in part by a Cottrell Scholarship awarded by the Research Corporation, by the MRSEC and CAREER programmes of the NSF and by DOE.

\section{Author contributions}

W.D.W., K.C. and M.C.B. shared equal responsibility for most aspects of this project from instrument construction to data collection and manuscript preparation. W.D.W. performed a majority of the analysis. T.K. grew the Bi-2201 samples and helped refine the STM. T.T. and H.I. contributed to Bi-2201 sample growth. Z.X., J.W. and G.D.G. contributed to Bi-2212 sample growth. Y.W. contributed to analysis and manuscript preparation. E.W.H. advised.

\section{Additional information}

Supplementary Information accompanies this paper on www.nature.com/naturephysics. Reprints and permissions information is available online at http://npg.nature.com/ reprintsandpermissions. Correspondence and requests for materials should be addressed to E.W.H. 